\title{
Evaluation of T2-W MR imaging and diffusion-weighted imaging for the early post-treatment local response assessment of patients treated conservatively for cervical cancer: a multicentre study
}

\author{
Maarten G. Thomeer ${ }^{1,2} \cdot$ Vincent Vandecaveye ${ }^{3} \cdot$ Loes Braun $^{1} \cdot$ Frenchey Mayer $^{4} \cdot$ Martine Franckena-Schouten $^{5}$. \\ Peter de Boer ${ }^{6}$. Jaap Stoker ${ }^{7} \cdot$ Erik Van Limbergen $^{8} \cdot$ Marrije Buist $^{9} \cdot$ Ignace Vergote $^{10} \cdot$ Myriam Hunink $^{1,11}$. \\ Helena van Doorn ${ }^{3}$
}

Received: 18 November 2017 / Revised: 28 March 2018 / Accepted: 20 April 2018 / Published online: 25 June 2018

(C) The Author(s) 2018

\begin{abstract}
Objectives To compare MR imaging with or without DWI and clinical response evaluation (CRE) in the local control evaluation of cervical carcinoma after radiotherapy.

Methods In a multicentre university setting, we prospectively included 107 patients with primary cervical cancer treated with radiotherapy. Sensitivity and specificity for CRE and MR imaging (with pre-therapy MR imaging as reference) (2 readers) were evaluated using cautious and strict criteria for identifying residual tumour. Nested logistic regression models were constructed for CRE, subsequently adding MR imaging with and without DWI as independent variables, as well as the pre- to post-treatment change in apparent diffusion coefficient (delta ADC).

Results Using cautious criteria, CRE and MR imaging with DWI (reader 1/reader 2) have comparable high specificity (83\% and 89\%/ 95\%, respectively), whereas MR imaging without DWI showed significantly lower specificity (63\%/53\%) than CRE. Using strict criteria, CRE and MR imaging with DWI both showed very high specificity (99\% and 92\%/95\%, respectively), whereas MR imaging without DWI showed significantly lower specificity (89\%/77\%) than CRE. All sensitivities were not significantly different. Addition of MR imaging with DWI to CRE has statistically significant incremental value in identifying residual tumour (reader 1: estimate, $1.06 ; p=$ 0.001) (reader 2: estimate, $0.62 ; p=0.02$ ). Adding the delta ADC did not have significant incremental value in detecting residual tumour. Conclusions DWI significantly increases the specificity of MR imaging in the detection of local residual tumour. Furthermore, MR imaging with DWI has significant incremental diagnostic value over CRE, whereas adding the delta ADC has no incremental diagnostic value.

Key Points

- If MR imaging is used for response evaluation, DWI should be incorporated

- MR imaging with DWI has diagnostic value comparable/complementary to clinical response evaluation

- Inter-reader agreement is moderate to fair for two experienced radiologist readers

- Quantitative measurements of ADC early post-therapy have limited diagnostic value
\end{abstract}

Maarten G. Thomeer

m.thomeer@erasmusmc.nl

1 Department of Radiology and Nuclear Medicine, Erasmus University Medical Center, Rotterdam, The Netherlands

2 Department of Radiology, Erasmus Medical Center Rotterdam, P.O Box 2040, 3015, CE Rotterdam, The Netherlands

3 Department of Radiology, University Hospitals Leuven, Leuven, Belgium

4 Department of Gynecology, Erasmus University Medical Center, Rotterdam, The Netherlands

5 Department of Radiotherapy, Erasmus University Medical Center, Rotterdam, The Netherlands
6 Department of Radiotherapy, Academic Medical Center, University of Amsterdam, Amsterdam, The Netherlands

7 Department of Radiology and Nuclear Medicine, Academic Medical Center, University of Amsterdam, Amsterdam, The Netherlands

8 Department of Radiotherapy, University Hospitals Leuven, Leuven, Belgium

9 Department of Gynecology Academic Medical Center, University of Amsterdam, Amsterdam, The Netherlands

10 Department of Gynecology, University Hospitals Leuven, Leuven, Belgium

11 Department of Epidemiology, Erasmus University Medical Center, Rotterdam, The Netherlands 
Keywords Uterine cervical neoplasm · Magnetic resonance imaging · Diffusion-weighted magnetic resonance imaging · Radiation · Comparative study

$\begin{array}{ll}\text { Abbreviations } \\ \text { ADC } & \text { Apparent diffusion coefficient } \\ \text { SADC } & \text { Change in apparent diffusion coefficient } \\ \text { CRE } & \text { Clinical response examination } \\ \text { DWI } & \text { Diffusion-weighted imaging } \\ \text { MR } & \text { Magnetic resonance } \\ \text { T2-WI } & \text { T2-weighted MR images }\end{array}$

\section{Introduction}

Cervical cancer represents a major health burden, with for instance around 12,000 newly diagnosed patients in the USA each year (US Cancer Statistics Working Group 2016). The standard treatment for locally advanced cervical cancer is external beam radiotherapy with image-guided adaptive brachytherapy $[1,2]$ and concurrent chemotherapy $[3,4]$ or hyperthermia [5]. More than $90 \%$ of patients show complete local response after such treatment [6-8].

To detect local residual tumour at an early time point (2-3 months after treatment) and allow potentially curative salvage therapy, response assessment after radiotherapy is uniformly recommended [9-11]. Although the effect of salvage surgery on survival is not well studied, retrospective studies suggest that early detection of local residual disease in asymptomatic women may offer survival benefit $[12,13]$. Moreover, salvage hysterectomy for local disease at a later, i.e. symptomatic, stage has high complication rates or is not feasible because of irresectable disease [14]. Therefore, a wellfounded diagnosis of local residual tumour shortly after completing radiotherapy would be helpful in the timely selection of women for salvage surgery, and might contribute to enhanced survival. Secondly, the patient with residual disease not suitable for salvage surgery might be quickly informed about her unfortunate prognosis in a timely manner.

In most centres, response assessment is performed via clinical response examination (CRE) at 2-3 months after radiotherapy $[1,2]$. This pelvic exam can be unsatisfactory because of the altered anatomy after radiotherapy (no cervix, stricture of the vagina, fibrosis) and the discomfort and pain experienced by patients. Subsequently an examination under anaesthesia may be necessary.

A seemingly attractive alternative after completing radiotherapy therefore is imaging, either with PET CT scanning or magnetic resonance (MR) imaging.

The use of PET-CT has recently been advised for response evaluation according to the National Comprehensive Cancer
Network (NCCN 2017). In the earlier European Society of Medical Oncology guideline PET-CT is not incorporated for response evaluation [15]. As far as we know, no data exists on its diagnostic value in the first response evaluation. For this reason we do not use PET-CT in our departments. Further prospective studies are needed to ensure its diagnostic value.

MR imaging has so far been unable to fulfil the need to detect residual disease after radiation because of a false positive rate of up to $45 \%[16,17]$ that is mainly attributable to difficulties in differentiating residual tumour from local oedema. The development of MR imaging with diffusion-weighted imaging (DWI) has led to investigations of its potential in differentiating high cellular matrix (as seen in tumour) from low cellular matrix (as seen in oedema). Several studies have shown promising results in identifying local residual tumour after radiotherapy, for instance in rectal cancer and breast cancer [18-21]. In 2015, a systematic review described the additional value of DWI in monitoring treatment response in patients undergoing chemoradiotherapy for locally advanced uterine cervical cancer [21]. A greater increase of the apparent diffusion coefficient (ADC) was seen after treatment versus before treatment if response was complete. However, data were not externally validated. Moreover, reference standard was mostly based on MR imaging findings. To the best of our knowledge, only two recent studies have evaluated the accuracy of MR imaging with DWI [22, 23]. The first study was a single-centre study of retrospective design, while the second study involved retrospective analysis without standard use of pathologic evidence as a reference standard, instead also using radiological follow-up.

The primary aim of our study was to perform a multicentre study of the incremental value of MR imaging with DWI compared to standard MR imaging in the first response evaluation after radiotherapy for cervical cancer. A secondary aim was to evaluate the additional value of MR imaging with or without DWI in comparison to CRE. Histopathology or a minimal follow-up of 1 year was used as reference standard.

\section{Materials and methods}

\section{Study design and patients}

This prospective study was approved by the institutional review board in the three participating university hospitals (Erasmus University Medical Centre Rotterdam, Academic 
Medical Centre Amsterdam, University Hospitals Leuven) and written informed consent was obtained from all patients. The study was registered under trial ID (NTR3345). Study reporting was in accordance with the Standards for Reporting Diagnostic Accuracy Studies (STARD 2015).

From March 2011 to April 2015, all patients of 18 years and older with a primary tumour of the cervix and scheduled for radiation therapy with curative intent were included. Pregnant women, women with a contraindication for general anaesthesia or MRI and women with cognitive deficits were excluded.

\section{Clinical response examination}

CRE was based on a pelvic exam, performed by a staff or fellow gynaecological oncologist. CRE included visualisation of the cervix and vagina with a speculum and bimanual rectovaginal palpation.

Treatment response was scored prospectively as either suspicious or not suspicious for residual tumour and was labelled 'indeterminate' when patients were too difficult to evaluate, when findings were equivocal, or when the patient refused evaluation at the outpatient clinic. The reader extracting the indeterminate results retrospectively from the medical records was blinded to the MR imaging findings.

For CRE, sensitivity and specificity were calculated on the basis of cautious criteria (i.e. indeterminate results were classified as residual tumour) and on strict criteria (i.e. indeterminate results were classified as complete response).

As a result of ethics requirements at one centre, the T2-W MRI alone was available to the treating clinicians after CRE. In no patient was the information from DWI used. Where CRE was negative and MR imaging findings without DWI were suspicious for residual tumour, a subsequent clinical examination under anaesthesia was performed or a closer follow-up proposed. Although this could induce verification bias in some cases, it probably had no influence on the reference standard result, since residual tumour would only be detected earlier than during follow-up. In no patient was the information of DWI used.

\section{MR imaging}

In each participating centre, at a predefined time point around 2 months after treatment, imaging was performed on a state-ofthe-art 3.0-Tesla MR scanner. In each participating centre, pretreatment MR imaging was done within 4 weeks of treatment start and response MR imaging was planned 6-8 weeks after finalising radiotherapy. The protocol differed slightly between different vendors (Philips, Best, the Netherlands and General Electrics, Milwaukee, WI). Protocols can be found in Table 1. Imaging was performed before start of treatment and after completing radiotherapy. One institution used intravaginal gel.

\section{MR image analysis}

MR imaging was reviewed independently by two experienced readers (reader 1, an abdominal radiologist for more than 14 years; reader 2, an abdominal radiologist for more than 10 years). Both readers were blinded to clinical and pathological results. The pre-therapy images were at the readers' disposal to correlate the location of the primary tumour, similar to the evaluation process performed in daily clinical practice.

Evaluation was based on first (1) T2-weighted MR images (T2-WI) in three directions, then (2) T2-WI with DWI, and finally (3) the change in apparent diffusion coefficient $(\delta \mathrm{ADC})$ between post-therapy and pre-therapy.

The post-therapy DWI was analysed first together with the information from before treatment. This is very helpful for localisation. The rationale for this was to imitate clinical routine as much as possible. We assume that in clinical practice all patients have an initial scan before therapy.

The criterion for residual tumour based on T2-WI was a solid residual mass with intermediate signal intensity. The criterion for residual tumour based on DWI was b1000 hyperintensity, not attributable to T2 shine-through, at the location of the primary tumour as correlated to the T2-WI. For the DWI evaluation, qualitative interpretation of the ADC map provided additional information when deemed necessary by the reader. For example, if the lesion had high intensity on DWI with a corresponding low value on the ADC map (based on qualitative interpretation without quantitative measurement), it was interpreted as tumour. In the third evaluation, based on objective quantitative measurement of the ADC values, the criterion for residual tumour was $\delta \mathrm{ADC} \leq 31.0 \times$ $10^{-4}$. As published earlier, a $\delta \mathrm{ADC} \geq 62.0 \times 10^{-4}$ was considered a complete response [21].

The ADC was measured by circular region of interest (ROI) as large as possible within the solid tumour on the ADC maps on the largest axial tumour slice. Special attention was paid to avoid the areas of focal signal intensity changes, susceptibility artefacts and necrosis. If during response evaluation no tumour was visible, the location of the tumour on the pre-therapy scan was used for ROI measurements. While the justification for this approach is debatable, in most previous studies on cervical carcinoma quantification has been performed in the same way, making it at least comparable [21].

The radiological response of the primary tumour was scored using a five-point score $(1=$ definite complete response, 2 = probable complete response, 3 = indeterminate result, 4 = probable residual tumour, $5=$ definite residual tumour). As for CRE, sensitivity and specificity were calculated on the basis of cautious criteria (i.e. indeterminate results were classified as residual tumour) and on strict criteria (i.e. indeterminate results were classified as complete response). 
Table 1 The protocol differed slightly between the different vendors (Philips Healthcare, Best, the Netherlands and General Electrics, Milwaukee, Wis). Imaging was performed before the start of treatment and after completing radiotherapy. One institution used intravaginal gel (Fig. 2b)

\begin{tabular}{llll}
\hline Scanner (3 Tesla) & & GE scanner (centre 1) & $\begin{array}{l}\text { Philips scanner } \\
\text { (centres 2 and 3) }\end{array}$ \\
\hline Patients included & & 85 & 22 \\
T2-weighting (2D) & TR/TE (ms/ms) & $9436 / 81$ & $4571 / 80$ \\
& Scan plane & 3 directions & 3 directions \\
& Flip angle (grades) & 120 & 90 \\
& Matrix size & $320 \times 320$ & $344 \times 285$ \\
& Bandwidth (kHz) & 125 & 125 \\
& Field view & 24 & 24 \\
& Number of excitations & 2 & 1 \\
& Slice thickness (mm) & 4 & 4 \\
& Gap & 0.4 & 0.4 \\
& Gradient & 3 directions & 3 directions \\
& Scan plane & Axial & Axial \\
& $b$ values & $0-50-100-500-750-1000$ & $0-50-100-500-750-1000$ \\
& Bandwidth (kHz) & 125 & 125 \\
& Slice thickness (mm) & 4 & 4 \\
\hline
\end{tabular}

\section{Reference standard}

All patients underwent a pelvic examination after radiotherapy and subsequently every 3 months, for at least a year. It is assumed that most small, clinically overt, residual tumours would become obvious within this follow-up period, although some recurrences can occur up to 3 years after therapy. Histopathological evaluation served as the reference standard in case of positive results. A recurrence-free follow-up period of at least 1 year was considered a surrogate reference standard for a complete local response. Routine histopathology in all cases would have added the burden of an additional examination under anaesthetic (EUA) and was deemed unacceptable.

\section{Statistical analysis}

For interpretation of T2-weighted images with or without DWI, interobserver agreement on the five-point scale was calculated via Cohen's kappa, using a weighted method based on the squared distance from the diagonal. Kappa values of 0 0.20 were interpreted as poor agreement, $0.21-0.40$ as fair, $0.41-0.60$ as moderate, $0.61-0.80$ as good and $0.81-1$ as excellent agreement.

A logistic regression model was constructed first with CRE only (model 1), and subsequently by adding T2-WI (model 2), T2-WI with DWI (model 3) and SADC (model 4) as independent variables for identification of residual tumour (dependent variable) by the two radiology readers. Our sample size calculation was based on optimising specificity, in order to decrease the false positive rate due to local oedema. We chose an alpha value of 0.05 and estimated the prevalence of residual tumour at $10 \%$. To demonstrate a significant difference in the expected specificity of 0.70 compared to the previously found specificity of 0.55 , with a power of 0.80 , would require 83 patients without residual tumour. With $10 \%$ expected to have residual tumour and $90 \%$ a complete response, 92 patients were required. Allowing for $10 \%$ loss to follow-up would mean that we needed 101 patients.

\section{Results}

\section{Patient and response characteristics}

A total of 133 patients were eligible; 26 patients were excluded [refusal of further inclusion $(n=17)$, change of therapy $(n=$ $5)$, further therapy elsewhere $(n=2)$, death during therapy $(n=$ $1)$, claustrophobia in MR scanner $(n=1)]$, leaving 107 patients for analyses. Baseline demographics and clinical characteristics are shown in Table 2 . The reason for the conservative treatment of the patient with a FIGO Ia was a relative contraindication for operation.

A complete response was found in 95 patients, whereas 12 patients $(11 \%)$ had local residual tumour on biopsy or resection (see examples in Figs. 1 and 2). The range of the tumour maximal diameter was between $1 \mathrm{~mm}$ and $62 \mathrm{~mm}$, with a mean of $24 \mathrm{~mm}$ and a standard deviation of $21 \mathrm{~mm}$. The mean time interval between the end of radiotherapy and response evaluation was 51 days (95\% CI 48-53) for CRE and 42 days (95\% CI 40-44) for MRI. Follow-up duration in patients without recurrence was between 1.1 and 5.2 years.

\section{Interobserver agreement}

Interobserver agreement on a five-point scale was fair (0.32) for interpretation of T2-WI and moderate $(0.46)$ for the interpretation of T2-WI + DWI. When the five-point scale was converted 
Table 2 Patient characteristics

\begin{tabular}{lll} 
Patient characteristics & & \\
Total number of patients & 107 & \\
Age (median, range) & $49(27-84)$ & $\%$ \\
Tumour stage & Number & 1 \\
FIGO & 1 & 4 \\
IA & 4 & 12 \\
IB1 & 13 & 5 \\
IB2 & 5 & 63 \\
IIA & 67 & 3 \\
IIB & 3 & 9 \\
IIIA & 10 & 4 \\
IIIB & 4 & \\
IVA & & 81 \\
Histologic subtype & 87 & 12 \\
Squamous cell carcinoma & 13 & 7 \\
Adenocarcinoma & 7 & 75 \\
Other & & 25 \\
Treatment & 80 & 98 \\
Chemoradiation & 27 & \\
Radiotherapy + hyperthermia & & \\
( Induction chemotherapy) & 105 & \\
Brachytherapy & 2 & \\
No brachytherapy & & \\
\hline
\end{tabular}

to a three-point scale, interobserver agreement was fair for interpretation of T2-WI (0.26) and T2-WI + DWI (0.39).

\section{Diagnostic performance of CRE and MR imaging}

Diagnostic performance in the interpretation of CRE, T2-WI and T2-WI + DWI are shown in Table 3. Almost all specificities of T2-WI with DWI (asterisk) were significantly higher compared to T2-WI. The sensitivities of T2-WI with DWI did not differ significantly compared to T2-WI.

Compared to CRE, MR imaging had significantly lower specificities in all situations (bold), and sensitivities and specificities of T2-WI with DWI were not significantly different. Only for reader 1 in the strict setting was sensitivity significantly higher than that for CRE (bold).

Logistic regression analyses of the effect of adding different MR imaging protocols to the CRE are shown in Table 4 . The model with only CRE (model 1) demonstrated the value of $\operatorname{CRE}(\beta=1.74, p=0.0006)$ in identifying residual tumour. Model 2 demonstrated the significant added value of T2-WI in addition to CRE (reader 1 : $\beta=$ $0.77, p=0.009$ ) (reader 2 : $\beta=0.88, p=0.01$ ). Adding DWI to the T2-WI protocol (model 3) demonstrated the significant added value of DWI (reader $1: \beta=1.06, p=$ 0.001 ) (reader $2: \beta=0.62, p=0.02$ ) and showed that T2WI with DWI can replace CRE. The addition of the $\delta$ ADC (model 4) had no added value for either reader.

\section{Difference in ADC between pre-treatment and response $M R I(\delta A D C)$}

We compared $\delta \mathrm{ADC}$ values for patients with a complete response and patients with residual tumour for both readers. According to the scatterplot depicting the $\delta \mathrm{ADC}$ values for both readers (Fig. 3), the values of the two readers were not comparable.

Further ROC analysis demonstrated moderate discrimination (Fig. 4): using the aforementioned cut-offs of $\geq 62 \times 10^{-4}$ for complete response and $\leq 31 \times 10^{-4}$ for residual tumour, the readers achieved only intermediate sensitivities and specificities.
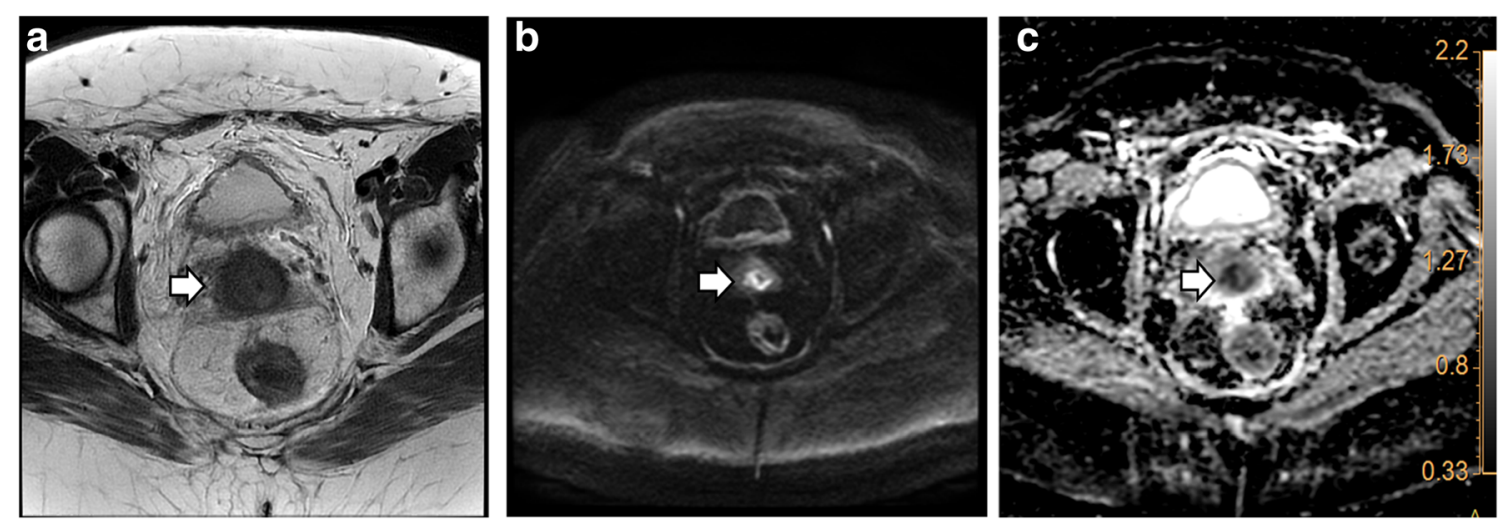

Fig. 1 This 50-year-old patient was clinically evaluated as non-suspect 61 days after radiation therapy. T2-weighted imaging (a, arrow) showed an indeterminate aspect of the cervix and was therefore supplemented with true cut biopsy under anaesthesia. This confirmed residual disease on histopathology. At the end of the study diffusion-weighted imaging (DWI) was analysed, blinded for the histopathology result. This showed (b, arrow) a clear hyperintense region. Visual ADC information (low intensity) was used to differentiate from mucosal oedema (c, arrow), leading to the diagnosis of residual tumour by both readers. The $\delta \mathrm{ADC}$ was only suspect for local residue for reader $2\left(\delta \mathrm{ADC}\right.$, reader $1: 52 \times 10^{-4}$ and reader 2: $29 \times 10^{-4}$ ) on the basis of aforementioned cut-off of $31 \times$ $10^{-4}$ 
Fig. 2 A 30-year-old patient with a normal post-therapeutic clinical examination. T2-weighted MR imaging (a, $\mathbf{b}$ arrow) showed an infracentimetric suspicious region centrally in the cervix. Local follow-up during 1 year remained normal. Diffusion-weighted imaging (DWI, c arrow) was not suspect for both readers. The ADC map (d arrow) was hyperintense (ADC, reader 1: $132 \times$ $10^{-4} \mathrm{~mm}^{2} / \mathrm{s}$ and reader $2: 125 \times$ $10^{-4} \mathrm{~mm}^{2} / \mathrm{s}$ ), confirming the presence of fluid rather than tumour. The delta ADC was not suspect for readers $(\delta \mathrm{ADC}$, reader 1: $63 \times 10^{-4}$ and reader 2: $52 \times$ $10^{-4}$ ) on the basis of aforementioned cut-off of $31 \times 10^{-4}$. Note the use of intravaginal gel in order to expand the vagina on $\mathbf{b}$ (white star)
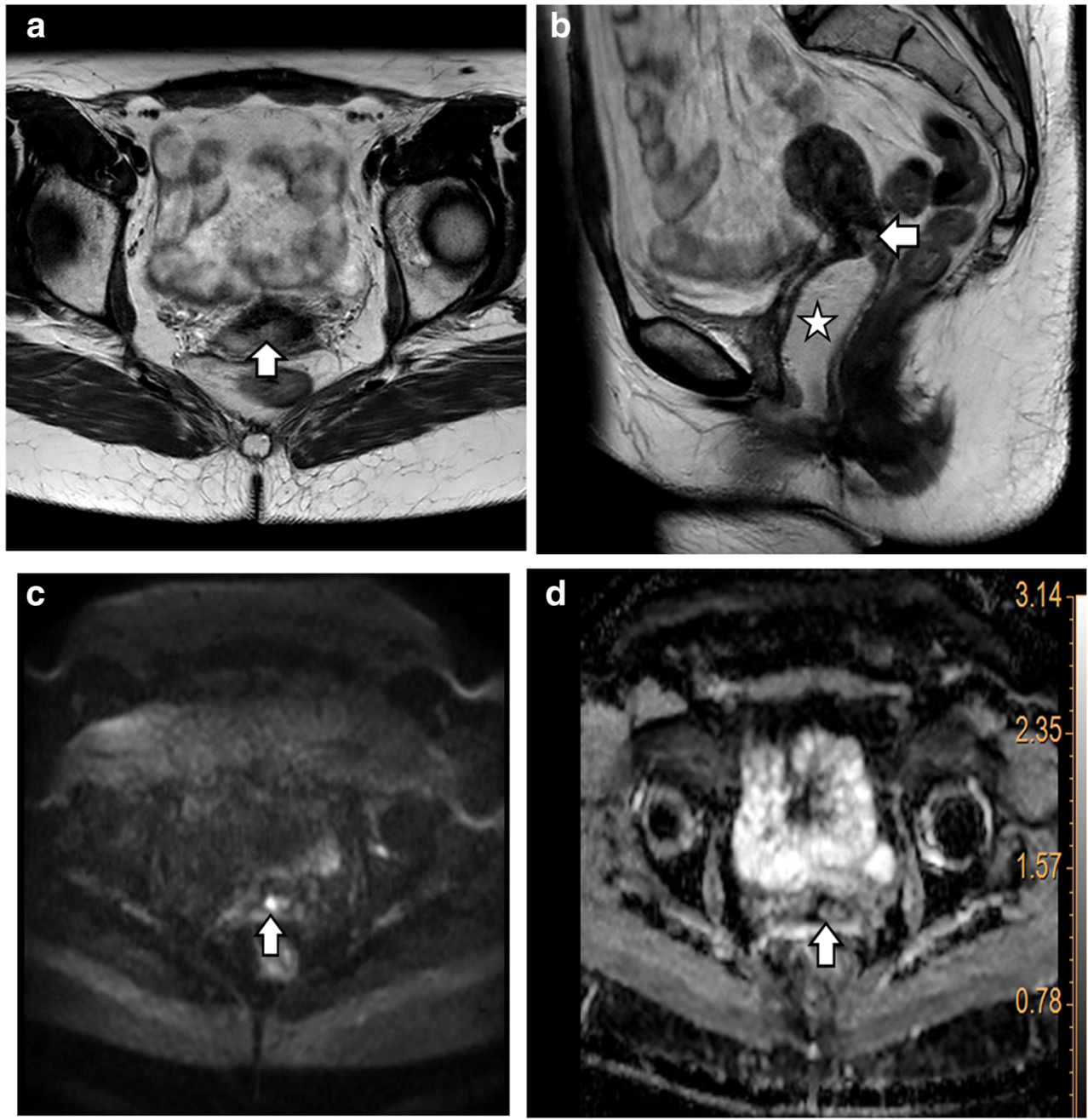

Table 3 CRE and MR imaging findings of local residual tumour with corresponding diagnostic values using cautious and strict criteria. Using cautious criteria indeterminate findings were labeled as suspicious for residual tumour and using strict criteria indeterminate findings were labeled as not suspicious for residual tumour. CRE clinical response examination, T2-WI T2-weighted MR imaging, T2-WI + DWI T2weighted imaging + diffusion-weighted imaging, CI 95\% confidence interval. Asterisk refers to the outcome which was significantly different on T2-WI + DWI compared to T2-WI. Bold highlights figures which were significantly different on T2-WI or T2-WI + DWI compared to CRE

\begin{tabular}{|c|c|c|c|c|c|}
\hline & & \multicolumn{2}{|c|}{ Cautions criteria } & \multicolumn{2}{|l|}{ Strict criteria } \\
\hline & & Sensitivity & Specificity & Sensitivity & Specificity \\
\hline \multirow[t]{2}{*}{ CRE } & & $58 \%(7 / 12)$ & $83 \%(79 / 95)$ & $25 \%(3 / 12)$ & $99 \%(94 / 95)$ \\
\hline & & (CI 32-82) & (CI 76-89) & (CI 7-53) & (CI 95-100) \\
\hline \multirow[t]{4}{*}{ T2-WI } & Reader 1 & $67 \%(8 / 12)$ & $63 \%(60 / 95)$ & $58 \%(7 / 12)$ & $89 \%(85 / 95)$ \\
\hline & & (CI 39-88) & (CI 54-71) & (CI 32-82) & (CI 83-94) \\
\hline & Reader 2 & $83 \%(10 / 12)$ & $53 \%(50 / 95)$ & $75 \%(9 / 12)$ & $77 \%(73 / 95)$ \\
\hline & & (CI 56-97) & (CI 44-61) & (CI 47-93) & (CI 69-84) \\
\hline \multirow[t]{4}{*}{ T2-WI+DWI } & Reader 1 & $83 \%(10 / 12)$ & $89 \%(85 / 95)^{*}$ & $83 \%(10 / 12)$ & $92 \%(87 / 95)$ \\
\hline & & (CI 56-97) & (CI 83-94) & (CI 56-97) & (CI 85-96) \\
\hline & Reader 2 & $50 \%(6 / 12)$ & $95 \%(90 / 95)^{*}$ & $50 \%(6 / 12)$ & $95 \%(90 / 95)^{*}$ \\
\hline & & (CI 25-75) & (CI 89-98) & (CI 25-75) & (CI 89-98) \\
\hline
\end{tabular}

\footnotetext{
*T2-WI with DWI statistically significantly higher compared to T2-WI
} 
Table 4 Logistic regression analyses of addition of various MR imaging protocols to the CRE. Parameters with significant $p$ values are shown in bold. CRE clinical response examination,

T2-WI T2-weighted MR imaging, T2-WI + DWI T2weighted imaging + diffusionweighted imaging, $\delta \mathrm{ADC}$ is the difference between the pretreatment $\mathrm{ADC}$ and the posttreatment $\mathrm{ADC}, \mathrm{ADC}$ apparent diffusion coefficient, estim. estimate, std err. standard error

\begin{tabular}{|c|c|c|c|c|c|c|c|}
\hline Reader 1 & & & & Reader 2 & & & \\
\hline \multirow[t]{2}{*}{ Model 1} & & & & Model 1 & & & \\
\hline & Estim. & Std. err. & $p$-value & & Estim. & Std. err. & $p$-value \\
\hline Intercept & -2.84 & 0.46 & $p<0.0001$ & Intercept & -2.84 & 0.46 & $p<0.0001$ \\
\hline CRE & 1.74 & 0.50 & 0.0006 & CRE & 1.74 & 0.51 & 0.0006 \\
\hline \multirow[t]{2}{*}{ Model 2} & & & & Model 2 & & & \\
\hline & Estim. & Std. err. & $p$-value & & Estim. & Std. err. & $p$-value \\
\hline Intercept & -4.61 & 0.95 & $p<0.0001$ & Intercept & -5.52 & 1.30 & $p<0.0001$ \\
\hline $\mathrm{CRE}$ & 1.22 & 0.58 & 0.03 & CRE & 1.47 & 0.57 & 0.01 \\
\hline MRimaging & 0.77 & 0.30 & 0.009 & MRimaging & 0.88 & 0.35 & 0.01 \\
\hline \multirow[t]{2}{*}{ Model 3} & & & & Model 3 & & & \\
\hline & Estim. & Std. err. & $p$-value & & Estim. & Std. err. & $p$-value \\
\hline Intercept & -5.43 & 1.17 & $p<0.0001$ & Intercept & -5.36 & 1.26 & $p<0.0001$ \\
\hline CRE & 1.08 & 0.65 & 0.09 & CRE & 1.03 & 0.66 & 0.1 \\
\hline MRimaging & 0.03 & 0.40 & 0.9 & MRimaging & 0.54 & 0.38 & 0.2 \\
\hline MRimaging+DWI & 1.06 & 0.32 & 0.001 & $\begin{array}{l}\text { MRimaging+ } \\
\text { DWI }\end{array}$ & 0.62 & 0.26 & 0.02 \\
\hline \multirow[t]{2}{*}{ Model 4} & & & & Model 4 & & & \\
\hline & Estim. & Std. err. & $p$-value & & Estim. & Std. err. & $p$-value \\
\hline Intercept & -7.09 & 2.35 & 0.003 & Intercept & -5.08 & 1.48 & 0.0006 \\
\hline CRE & 1.35 & 0.77 & 0.07 & CRE & 0.96 & 0.69 & 0.2 \\
\hline MRimaging & 0.10 & 0.41 & 0.8 & MRimaging & 0.56 & 0.39 & 0.2 \\
\hline MRimaging+DWI & 1.29 & 0.43 & 0.003 & $\begin{array}{l}\text { MRimaging+ } \\
\text { DWI }\end{array}$ & 0.59 & 0.29 & 0.04 \\
\hline$\delta A D C$ & 0.01 & 0.02 & 0.4 & $\delta A D C$ & -0.00 & 0.01 & 0.7 \\
\hline
\end{tabular}

\section{Discussion}

In this prospective multicentre study, we found that DWI significantly increases the specificity of MR imaging in the detection of residual local tumour compared to $\mathrm{T} 2 \mathrm{~W}$ imaging alone during response evaluation of cervical carcinoma following radiotherapy. MR imaging with DWI also showed significant incremental value over CRE.

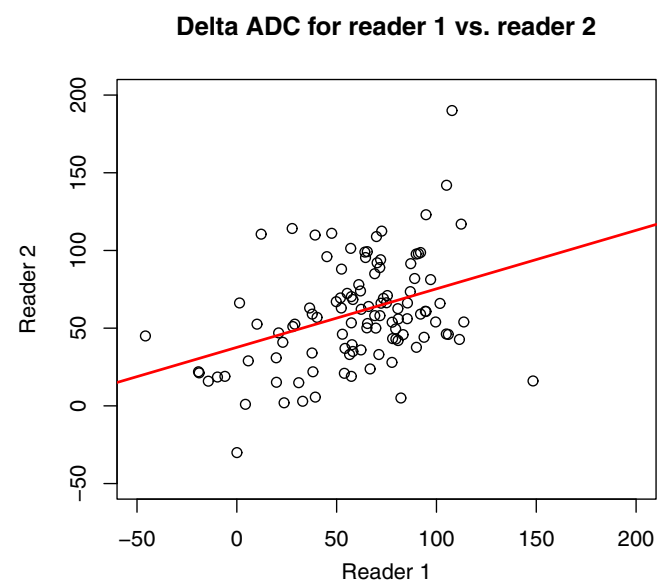

Fig. 3 Scatter plot comparing delta ADC values for reader 1 and reader 2 shows the values were not comparable. ADC apparent diffusion coefficient
In a situation where MR imaging is appended to the treatment response evaluation, DWI information should always be added to reduce the chance of incorrectly declaring an unfavourable outcome, i.e. presumed residual tumour in cases with a complete response. This is in line with our hypothesis, since the presence

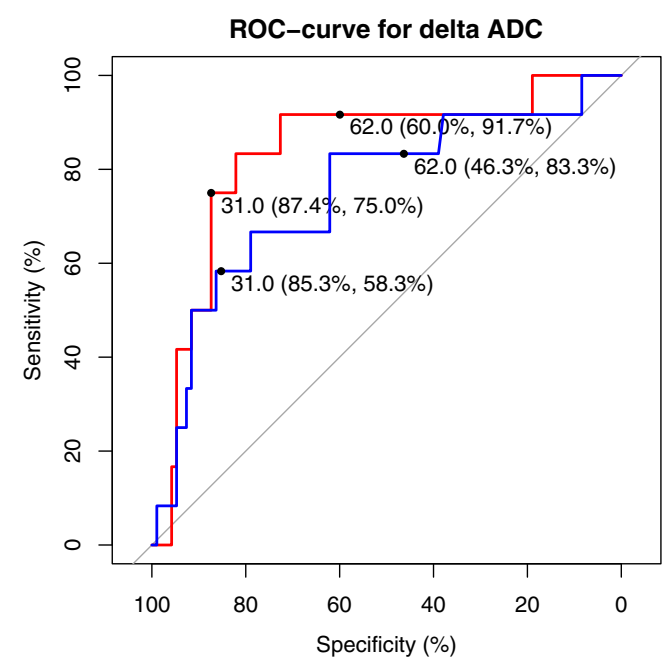

Fig. 4 ROC analysis of change of ADC between pre- and post-treatment $\mathrm{ADC}(\delta \mathrm{ADC})$ for reader 1 (red line) and reader 2 (blue line). Cut-offs for complete response $\left(\geq 62 \times 10^{-4}\right)$ and residual tumour $\left(\leq 31 \times 10^{-4}\right)$ and their corresponding sensitivities and specificities are shown 
of oedema hampers T2-WI, resulting in misdiagnoses of residual disease. Oedema is typically seen in the first months after radiation therapy, at the time when response evaluation is performed to decide on complementary surgery in case of residual disease. Our finding is also in line with a previous study showing that T2-WI alone resulted in up to $50 \%$ false positives [16].

Our findings are in accordance with a recent retrospective study, where a high specificity ( $84 \%$ ) was found using a combination of high intensity on T2-WI and high intensity on DWI [22]. However, that study differed from ours since their inclusion consisted mainly of lower stages of cervical carcinomas in a neoadjuvant setting.

To the best of our knowledge, our study is the first to compare CRE with combined T2-WI and DWI for early response assessment after radiation for cervical carcinoma. One study evaluating CRE versus conventional MR imaging in the response assessment after radiation therapy reported that MRI scored significantly worse than CRE, mainly in terms of specificity [16]. However, as that study did not incorporate DWI, it is difficult to compare their results with ours. With the current protocol, we found that MR imaging with DWI significantly improves work-up compared to CRE. It is unlikely that there was bias from the same clinician doing the clinical assessment as doing the CRE, as these assessments were done a year apart.

One could argue that as a result of current optimised radiation therapy schemes, including MR imaging or CT-guided brachytherapy, the percentage of patients with local residual tumour after radiotherapy treatment for cervical cancer is too low to justify further protocols such as MR imaging. Distant recurrence probably has more impact on survival. However, it remains a fact that only local residual tumour can potentially be cured, for instance with salvage surgery. Despite the low prevalence of local residual tumour without distant metastases, it is therefore imperative that the patient with residual tumour is recognised soon after treatment while salvage is still an option.

Logistic regression analysis showed that if both CRE and MR imaging with DWI are used, only the latter has a significant impact on identifying local residual tumour. A first option would be to replace all CRE by MR imaging with DWI. Although theoretically our data suggest that this would be an acceptable alternative, in clinical practice clinicians would still prefer to examine the patient during the first response evaluation, since it is inexpensive. Moreover, very small superficial and mucosal based residual tumours will probably remain undetected with MR imaging. A second option could be to use MR imaging with DWI in case CRE is difficult to perform or when the patient is uncooperative. A third option is to perform both CRE and MR imaging during the first response evaluation in all cases. The last two options have four clear advantages. First, the MR images can be reviewed at a later time point and can be discussed with colleagues, which is impossible with CRE. CRE also depends on the experience of the physician. A second advantage is that these initial MRI data are available during follow-up, particularly in cases in which new (clinical) problems and queries arise. The third advantage of using MR imaging is to guide eventual biopsy more precisely in the deeper cervical regions that are more difficult to evaluate with CRE. Finally, in the case of proven local residual tumour, MR imaging is usually better than CRE in identifying local parametrial or pelvic wall invasion, which could make salvage hysterectomy unfeasible, as was shown in an earlier meta-analysis by our study group [24].

A final option is to add MR imaging depending on the probability of local residual tumour and distal tumour recurrence. Higher stages of cervical carcinoma, especially those with possible lymphadenopathy outside the radiation field, would have less benefit of salvage surgery, making MRI with DWI less useful.

Quantitative evaluation of the ADC post-therapy versus pretherapy was less helpful in our study, in contrast to findings reported in an earlier systematic review [21]. The overall absolute cut-offs found for residual tumour versus absence thereof were not reproduced in our multicentre setting. The use of different MR systems may have possibly played a role in our varied results. Indeed, a previous study has shown that absolute $\mathrm{ADC}$ values can be vendor-specific, depending on the region scanned in the upper abdomen [25]. As far as we know, the reproducibility of ADC values in the cervical regions across different scanners has not been fully studied. However, in terms of our findings, it is debatable whether the use of different vendors explains the differences, since only two protocols were used, with very similar sequence details and $b$ values. On the basis of our findings, we conclude that measurement of ADC differences is time-consuming and is not particularly helpful, even more so because our logistic regression model showed no influence of these data on the final diagnosis of local residual tumour for either radiologist reader.

There are some drawbacks to our study. The inter-reader agreement was only moderate to fair for MR imaging evaluation, which might suggest that the results are not fully reproducible. This could be an important obstacle to further implementation of this technique. However, the readers took different approaches to image evaluation despite having received the same predetermined interpretation criteria as mentioned earlier. Reader 1 was more cautious and evaluated indeterminate lesions more often as residual tumour, thereby increasing sensitivity, while reader 2 used more strict criteria for diagnosing residual tumour, i.e. he evaluated indeterminate lesions more often as a complete response. Furthermore, reader 1 had more experience with DWI in the pelvis (10 years versus 6 years), which could also explain his higher sensitivity (although not significantly higher). Further studies should be performed to establish the reproducibility of DWI for identifying residual cervical cancer post radiotherapy.

The results concerning sensitivity were not significantly different across protocols. As a result of the low prevalence of residual disease, our study would have required over 240 patients to allow conclusions about sensitivity. For reasons of feasibility we powered the study to demonstrate an increase in specificity by adding DWI to the T2-weighted imaging. 
According to the most recent guidelines, treatment evaluation should be done around 3 months after the end of treatment [15]. We decided to perform the MRI some weeks earlier, mainly because we wanted to diagnose residual tumour as soon as possible, in order to enhance the possibilities of local salvage. Too soon after treatment though, the interpretation could be hampered by oedema. However, we prioritised early detection, as the use of DWI for identifying tumour is not compromised by local oedema.

In conclusion, the addition of DWI to the standard MRI protocol significantly improves the value of MR imaging. Furthermore, MR imaging with DWI has significant incremental value relative to CRE in finding residual cervical tumour after radiation therapy, and could possibly replace the latter in situations where CRE is less practical. ADC has no incremental diagnostic value.

Acknowledgements We would like to acknowledge the staff of the trial office of the Department of Radiology of Erasmus MC, particularly Laurens Groenendijk and Caroline Van Bavel. Without their daily help in data management this paper would not have been possible. Also, special gratitude to Willemien Hompus for her very helpful assistance during the inclusions.

Funding This study has received funding by Fonds Nuts Ohra.

\section{Compliance with ethical standards}

Guarantor The scientific guarantor of this publication is Thomeer Maarten

Conflict of interest The authors of this manuscript declare no relationships with any companies whose products or services may be related to the subject matter of the article.

Statistics and biometry Two of the authors have significant statistical expertise (Myriam Hunink and Loes Braun).

Informed consent Written informed consent was obtained from all subjects (patients) in this study.

Ethical approval Institutional review board approval was obtained.

\author{
Methodology \\ - prospective \\ - diagnostic or prognostic study \\ - multicentre study
}

Open Access This article is distributed under the terms of the Creative Commons Attribution 4.0 International License (http:// creativecommons.org/licenses/by/4.0/), which permits unrestricted use, distribution, and reproduction in any medium, provided you give appropriate credit to the original author(s) and the source, provide a link to the Creative Commons license, and indicate if changes were made.

\section{References}

1. Hellebust TP, Kirisits C, Berger D et al (2010) Recommendations from Gynaecological (GYN) GEC-ESTRO Working Group: considerations and pitfalls in commissioning and applicator reconstruction in 3D image-based treatment planning of cervix cancer brachytherapy. Radiother Oncol 96:153-160

2. Gaffney DK, Erickson-Wittmann BA, Jhingran A et al (2011) ACR Appropriateness Criteria(R) on Advanced Cervical Cancer Expert Panel on Radiation Oncology-Gynecology. Int J Radiat Oncol Biol Phys 81:609-614

3. Vale CL, Tierney JF, Davidson SE, Drinkwater KJ, Symonds P (2010) Substantial improvement in UK cervical cancer survival with chemoradiotherapy: results of a Royal College of Radiologists' audit. Clin Oncol (R Coll Radiol) 22:590-601

4. Green J, Kirwan J, Tierney J et al (2005) Concomitant chemotherapy and radiation therapy for cancer of the uterine cervix. Cochrane Database Syst Rev. https://doi.org/10.1002/14651858.CD002225. pub2:CD002225

5. Lutgens L, van der Zee J, Pijls-Johannesma M et al (2010) Combined use of hyperthermia and radiation therapy for treating locally advanced cervical carcinoma. Cochrane Database Syst Rev. https://doi.org/10.1002/14651858.CD006377.pub2:CD006377

6. Lindegaard JC, Fokdal LU, Nielsen SK, Juul-Christensen J, Tanderup K (2013) MRI-guided adaptive radiotherapy in locally advanced cervical cancer from a Nordic perspective. Acta Oncol 52:1510-1519

7. Potter R, Georg P, Dimopoulos JC et al (2011) Clinical outcome of protocol based image (MRI) guided adaptive brachytherapy combined with 3D conformal radiotherapy with or without chemotherapy in patients with locally advanced cervical cancer. Radiother Oncol 100:116-123

8. Hellebust TP, Kristensen GB, Olsen DR (2010) Late effects after radiotherapy for locally advanced cervical cancer: comparison of two brachytherapy schedules and effect of dose delivered weekly. Int J Radiat Oncol Biol Phys 76:713-718

9. Committee on Practice B-G (2002) ACOG practice bulletin. Diagnosis and treatment of cervical carcinomas, number 35 , May 2002. Obstet Gynecol 99:855-867

10. Elit L, Kennedy EB, Fyles A, Metser U (2016) Follow-up for cervical cancer: a program in evidence-based care systematic review and clinical practice guideline update. Curr Oncol 23:109-118

11. Elit L, Fyles AW, Devries MC, Oliver TK, Fung-Kee-Fung M, Gynecology Cancer Disease Site Group (2009) Follow-up for women after treatment for cervical cancer: a systematic review. Gynecol Oncol 114:528-535

12. Bodurka-Bevers D, Morris M, Eifel PJ et al (2000) Posttherapy surveillance of women with cervical cancer: an outcomes analysis. Gynecol Oncol 78:187-193

13. Chou HH, Wang CC, Lai CH et al (2001) Isolated paraaortic lymph node recurrence after definitive irradiation for cervical carcinoma. Int J Radiat Oncol Biol Phys 51:442-448

14. Sardain H, Lavoue V, Redpath M, Bertheuil N, Foucher F, Leveque $\mathrm{J}$ (2015) Curative pelvic exenteration for recurrent cervical carcino$\mathrm{ma}$ in the era of concurrent chemotherapy and radiation therapy. A systematic review. Eur J Surg Oncol 41:975-985

15. Colombo N, Carinelli S, Colombo A et al (2012) Cervical cancer: ESMO Clinical Practice Guidelines for diagnosis, treatment and follow-up. Ann Oncol 23 Suppl 7:vii27-vii32

16. Vincens E, Balleyguier C, Rey A et al (2008) Accuracy of magnetic resonance imaging in predicting residual disease in patients treated for stage IB2/II cervical carcinoma with chemoradiation therapy : correlation of radiologic findings with surgicopathologic results. Cancer 113:2158-2165

17. Hequet D, Marchand E, Place V et al (2013) Evaluation and impact of residual disease in locally advanced cervical cancer after concurrent chemoradiation therapy: results of a multicenter study. Eur J Surg Oncol 39:1428-1434

18. Kim SH, Lee JM, Hong SH et al (2009) Locally advanced rectal cancer: added value of diffusion-weighted MR imaging in the 
evaluation of tumor response to neoadjuvant chemo- and radiation therapy. Radiology 253:116-125

19. Lambregts DM, Vandecaveye V, Barbaro B et al (2011) Diffusionweighted MRI for selection of complete responders after chemoradiation for locally advanced rectal cancer: a multicenter study. Ann Surg Oncol 18:2224-2231

20. Bufi E, Belli P, Costantini M et al (2015) Role of the apparent diffusion coefficient in the prediction of response to neoadjuvant chemotherapy in patients with locally advanced breast cancer. Clin Breast Cancer 15:370-380

21. Schreuder SM, Lensing R, Stoker J, Bipat S (2015) Monitoring treatment response in patients undergoing chemoradiotherapy for locally advanced uterine cervical cancer by additional diffusionweighted imaging: a systematic review. J Magn Reson Imaging 42:572-594

22. Jalaguier-Coudray A, Villard-Mahjoub R, Delouche A et al (2017) Value of dynamic contrast-enhanced and diffusion-weighted MR imaging in the detection of pathologic complete response in cervical cancer after neoadjuvant therapy: a retrospective observational study. Radiology. https://doi.org/10.1148/radiol.2017161299: 161299

23. Park JJ, Kim CK, Park BK (2016) Prediction of disease progression following concurrent chemoradiotherapy for uterine cervical cancer: value of post-treatment diffusion-weighted imaging. Eur Radiol 26:3272-3279

24. Thomeer MG, Gerestein C, Spronk S, van Doorn HC, van der Ham E, Hunink MG (2013) Clinical examination versus magnetic resonance imaging in the pretreatment staging of cervical carcinoma: systematic review and meta-analysis. Eur Radiol 23:2005-2018

25. Donati OF, Chong D, Nanz D et al (2014) Diffusion-weighted MR imaging of upper abdominal organs: field strength and intervendor variability of apparent diffusion coefficients. Radiology 270:454 463 